\title{
La génomique nutritionnelle : (re)penser les liens alimentation-santé à l'articulation des sciences sociales, biomédicales et de la vie
}

\author{
Tristan Fournier ${ }^{1, *}$ et Jean-Pierre Poulain ${ }^{2, a}$ \\ 1 Sociologue, CNRS, Iris (UMR 8156 CNRS - EHESS - U997 Inserm - UP13), Paris, France \\ ${ }^{2}$ Sociologue, Université de Toulouse Jean Jaurès, Certop (UMR 5044 CNRS), Toulouse, France
}

Reçu le 3 février 2016. Accepté le 2 mai 2017

Les progrès de la génomique n'ont pas permis de tenir les promesses que certains mettaient en elle. Le développement de l'épigénétique met de plus en plus en évidence le fait que génome et environnement interagissent de manière complexe. Dans le domaine de l'alimentation sur lequel se penche cet article, cela conduit à envisager des formes de prévention par une conduite personnalisée des pratiques individuelles. L'intérêt de cet article n'est pas seulement de souligner les enjeux politiques et sociaux de cette perspective en termes d'individualisation des responsabilités et donc de désocialisation du manger, il est aussi de montrer la nécessité d'une approche conjointe par les disciplines des sciences sociales et des sciences biologiques dans l'analyse des données qu'elles accumulent sur ces questions. Cela implique, pour ses auteurs, de mettre en cause l'autonomie tant du social que du biologique.

La Rédaction

\begin{abstract}
Résumé - Cet article étudie d'un point de vue sociologique l'émergence de la génomique nutritionnelle, thématique scientifique née du Projet Génome Humain qui ambitionne de renouveler le diagnostic, la prévention et la prise en charge des pathologies chroniques dans lesquelles l'alimentation serait impliquée. Souvent présentée comme une révolution scientifique, elle permettrait de formuler avec plus de précision les facteurs de risque associés à chaque patrimoine (épi)génétique et donc d'envisager des recommandations nutritionnelles personnalisées. Après avoir défini ce domaine de recherches, l'article en présente les principaux enjeux (émergence de nouvelles formes de responsabilité et perturbation des modes de régulation sociale de l'alimentation) et tente de voir dans quelle mesure la génomique nutritionnelle contribue à reproblématiser le dialogue entre les sciences sociales, biomédicales et de la vie autour des liens alimentationsanté.
\end{abstract}

Mots-clés : environnement / épigénétique / interdisciplinarité / nutrigénétique / santé

\begin{abstract}
Nutritional (epi)genomics: (re)considering the food-health relationships by connecting social, biomedical and life sciences. This article investigates, from a sociological perspective, the emergence of nutritional (epi)genomics, a field of research that has emerged from the Human Genome Project and whose aim is to renew the diagnosis, prevention and management of foodrelated chronic pathologies. By identifying more precisely the genetic and epigenetic risk factors at the individual level, this would indeed lead to the development of personalized nutrition. As such, it is often presented as a scientific revolution. The first part of the paper defines this research field by discussing its main scientific issues and presenting its medical applications that consist in biotechnology which has been available on the market over the past decade (nutrigenetic testing) as well as a public health program which is currently being drawn up (the "first 1000 days" initiative). Then it focuses on its social, ethical and political consequences as it could induce new forms of responsibility at the individual, intergenerational and
\end{abstract}

\footnotetext{
${ }^{a}$ J.-P. Poulain est professeur de sociologie et titulaire de la «Chair of Food Studies: Food, Cultures and Health», créée conjointement par l'Université de Toulouse Jean Jaurès et la Taylor's University de Kuala Lumpur (Malaysia). Il co-dirige le LIA-CNRS «Alimentation, Cultures et Santé».

${ }^{*}$ Auteur correspondant : tristan.fournier@ehess.fr
} 
political scales, and also heighten the medicalization of food and, consequently, its individualization. Finally the paper tries to understand to what extent the field of nutritional (epi)genomics may contribute to (re) consider the dialogue between social, biomedical and life sciences regarding the food and health relationships.

Keywords: environment / epigenetics / health / interdisciplinarity / nutrigenomics

En 2003, la fin du séquençage du génome humain s'est accompagnée d'une importante désillusion scientifique $^{1}$. Il s'est en effet révélé beaucoup plus difficile que ne le pensaient les chercheurs et leurs bailleurs de fond de passer de la cartographie génomique à des applications thérapeutiques concrètes, alors que celles-ci avaient constitué l'un des arguments justifiant des investissements records. Au-delà de la déception, le séquençage a toutefois permis de commencer à développer une médecine dite "personnalisée » dont l'une des applications, la génomique nutritionnelle $(\mathrm{GN})$, se concentre sur l'étude des interactions gènes-environnement et ambitionne de renouveler le diagnostic, la prévention et la prise en charge des pathologies chroniques dans lesquelles l'alimentation serait impliquée. La GN est souvent présentée comme une révolution scientifique car elle permettrait d'identifier avec plus de précision les facteurs de risque associés à chaque patrimoine (épi) génétique et donc d'envisager des recommandations nutritionnelles adaptées à un individu particulier.

Cet article poursuit trois objectifs : il vise d'abord à présenter et définir le domaine émergeant de la GN, il discute ensuite les principaux enjeux éthiques, sociaux et sanitaires liés à la personnalisation de la médecine et de la nutrition, et il tente enfin de voir comment ces développements scientifiques contribuent à re-problématiser le dialogue entre les sciences sociales, biomédicales et de la vie autour des liens alimentation-santé.

D'un point de vue théorique, l'article s'inscrit à la croisée de plusieurs champs sociologiques. D'abord celui de la sociologie de l'alimentation, qui analyse dans une perspective critique la montée en charge de la problématique nutritionnelle et sa mise en concurrence avec les autres dimensions de l'acte alimentaire, notamment la socialité (Poulain, 2001; Fournier, 2014). La sociologie des sciences est ensuite mobilisée à deux titres : d'une part pour interroger les possibilités d'interdisciplinarité, en particulier les conditions de mise en œuvre de recherches intersectorielles (Vinck, 2000 ;

\footnotetext{
${ }^{1}$ Le séquençage du génome humain n'a permis d'identifier que quelques 23000 gènes contre les 100000 attendus. Qui plus est, il est apparu que ces gènes étaient apparentés aux gènes déjà existants chez d'autres espèces alors que l'on pensait découvrir des gènes qui auraient permis de comprendre le phénomène humain (Barnes et Dupré, 2008).
}

Prud'homme et Gingras, 2015), et d'autre part pour documenter le processus de thématisation de ce nouvel objet scientifique. L'étude en termes de thématisation s'inscrit dans une tradition fondée en épistémologie par Gerald Holton (1982) et développée en sociologie par Jean-Michel Berthelot (1996). Elle consiste à s'intéresser aux processus scientifiques qui contribuent à l'organisation cognitive d'un objet. On peut distinguer deux versants du processus de thématisation: l'un interne au champ scientifique et l'autre à l'interface science-société. Dans le premier, il est à l'œuvre dans le cadre des débats, des concurrences paradigmatiques et des controverses. Dans le second, il résulte des interactions entre les acteurs scientifiques, les bailleurs de fonds publics ou privés, les utilisateurs potentiels des découvertes et enfin les acteurs du monde médiatique qui contribuent à la légitimité et à la notoriété des chercheurs et de ceux qui les supportent. Le matériau empirique qui permet d'étudier ce «travail d'organisation ${ }^{2}$ cognitive comprend les ouvrages et articles scientifiques, et les colloques, congrès et séminaires au cours desquels se présentent des résultats et se discutent des interprétations et argumentations. C'est dans ces " arènes scientifiques » que se met en place l'organisation problématique d'un objet scientifique et que s'opère l'articulation entre les savoirs établis et les nouveaux qui viennent les compléter, les prolonger ou les remettre en cause. La thématisation a également un autre versant: celui des «arènes sociales» dans lesquelles le champ scientifique entre en interaction avec les acteurs privés et publics des différents secteurs économiques et sociaux intéressés par l'application de ces découvertes scientifiques, les médias qui vont rendre compte de ces avancées et analyser leurs enjeux, les politiques qui vont règlementer et encadrer, les associations et organisations non gouvernementales et enfin les consommateurs et citoyens. Ces interactions et les logiques croisées d'intérêt qui les sous-tendent agissent également sur la thématisation scientifique en la dynamisant ou en la ralentissant, en donnant des justifications à certains choix d'application ou au contraire en pointant leurs risques ou leurs contradictions avec des principes, par exemple éthiques. Il est donc possible de distinguer deux espaces de thématisation qui

\footnotetext{
${ }^{2}$ Expression empruntée à Gilbert de Terssac et Karine Lalande (2002) et déplacée du champ de l'organisation du travail à celui de la sociologie des sciences.
} 
Tab. 1. Nutri-génétique et nutri-épigénétique.

Source: les auteurs

\begin{tabular}{|c|c|c|}
\hline & Nutri-génétique et nutri-génomique & Nutri-épigénétique \\
\hline Définitions & $\begin{array}{l}\text { Étude des interactions entre les gènes et les } \\
\text { nutriments, ainsi que des effets de ces } \\
\text { interactions sur la santé. }\end{array}$ & $\begin{array}{l}\text { Étude de l'impact des facteurs nutritionnels sur } \\
\text { l'expression des gènes, par une modification } \\
\text { temporaire inscrite sur la molécule d'ADN mais } \\
\text { sans modification de la séquence. }\end{array}$ \\
\hline État des connaissances & $\begin{array}{l}\text { Identification des effets d'interaction entre } \\
\text { des nutriments et les variations génétiques } \\
\text { de certains gènes. } \\
\text { Le domaine des risques cardio-vasculaires } \\
\text { est le plus investi. }\end{array}$ & $\begin{array}{l}\text { Réversibilité et transmissibilité (partiellement) } \\
\text { des marques épigénétiques induites par } \\
\text { l'alimentation. } \\
\text { Les mille premiers jours de vie comme fenêtre } \\
\text { d'exposition critique. }\end{array}$ \\
\hline Applications existantes & $\begin{array}{l}\text { Une application biotechnologique : les tests } \\
\text { nutri-génétiques. }\end{array}$ & $\begin{array}{l}\text { Un programme de santé publique: «l'initiative } \\
\text { des mille jours }{ }^{5} \text {. }\end{array}$ \\
\hline
\end{tabular}

se nourrissent l'un l'autre. Les travaux sur l'obésité ou sur les food studies de Jean-PierrePoulain $(2009$; 2017), sur le cholestérol de Tristan Fournier (2012b), sur la naturalité d'Olivier Lepiller (2012) ou sur le plaisir alimentaire d'Anne Dupuy (2014) s'inscrivent dans cette perspective.

Cet article explore la thématisation de la GN dans ces arènes scientifique et sociale. Les éléments exposés et discutés ici sont issus d'un programme de recherche ${ }^{3}$ au sein duquel plusieurs outils méthodologiques ont été mobilisés. Une première phase de veille scientifique visait à suivre l'activité scientifique de la GN afin d'en repérer les étapes de problématisation, d'en saisir les champs d'application, d'identifier les chercheurs impliqués (discipline, tutelle, localité) et de caractériser les espaces de publication (par exemple les types de revues et congrès). En complément d'une recherche documentaire couplée à une alerte électronique active depuis 2013, une revue de littérature systématique (PubMed, Web of Science et Francis) a été réalisée : 79 articles scientifiques, ouvrages et chapitres d'ouvrage rédigés en français et en anglais ont ainsi été étudiés. Puis des entretiens d'experts (au nombre de 13) ont été réalisés avec des représentants de différentes disciplines scientifiques (biologie, droit, génétique, nutrition, pharmacologie et sociologie). Enfin, la participation à 9 congrès, conférences et journées d'étude en France, en Europe et au Canada ( 6 centrés exclusivement sur la GN et 3 dans lesquels elle était une question importante) a permis aux auteurs, à travers leurs statuts d'auditeurs (dans 5 cas) et d'orateurs (dans 4 cas), d'adopter des postures d'obser-

\footnotetext{
$\overline{{ }^{3} \text { Le programme de recherche }}$ «NutriGen - Enjeux sociaux et scientifiques d'une nutrition personnalisée »- a été financé par le CNIEL-OCHA (Centre national interprofessionnel de l'économie laitière - Observatoire CNIEL des habitudes alimentaires) pour une durée de deux ans (2013-2015). Les auteurs déclarent n'avoir aucun conflit d'intérêt.
}

vation et d'observation participante pour analyser les points de débat et les controverses qui animent ce domaine de recherches, et d'identifier les différents camps scientifiques et leurs arguments propres. L'ambition de cet article est de rendre compte de l'émergence de la GN et de documenter le processus de thématisation de cet espace scientifique en repérant les enjeux épistémologiques et sociaux qui l'accompagnent.

\section{Nutrition, génétique et épigénétique}

Trois thématiques interdépendantes composent la GN. Les deux premières - la nutri-génétique et la nutrigénomique - constituent des champs de recherche à part entière donnant déjà lieu à des applications biomédicales; elles se distinguent de la troisième - la nutriépigénétique - plus récente, et qui vient non seulement les complexifier mais aussi redessiner le territoire scientifique (Tab. 1).

\section{Nutri-génétique et nutri-génomique: une vision déterministe de la santé humaine}

La nutri-génétique et la nutri-génomique désignent, respectivement ${ }^{4}$, l'étude des influences des gènes sur les réponses aux pratiques alimentaires, et celle des

\footnotetext{
${ }^{4}$ Même si des controverses scientifiques persistent quant aux frontières entre les deux champs, notamment dans leur définition respective, il est généralement admis que la nutrigénomique comprend la nutri-génétique. Dans la littérature anglo-saxonne, le terme «nutrigenomics» est utilisé pour parler des deux thématiques.

${ }^{5}$ Un test épigénétique du cancer de la prostate a également vu le jour en 2014, commercialisé par l'entreprise MDXHealth : http://mdxhealth.com/products-and-technology/products/con firmmdx-for-prostate-cancer
} 
Tab. 2. Marché et régulation des tests nutri-génétiques.

Source: les auteurs

\section{Le marché des tests nutri-génétiques}

2001 : l'entreprise anglaise Sciona commercialise les premiers tests par internet et par le biais d'une chaîne de magasins (The Body Shop). Après destruction de l'un d'eux par une association de consommateurs, elle se délocalise aux USA.

Prix : $200 \$$

2007 : publicités dans les espaces publics (Australie, Canada et USA).

2008 : 9 entreprises commercialisent des tests (dont 8 aux USA).

2013 : 56 entreprises commercialisent des tests. Premier prix : $99 \$$

2014 : 23andme, considéré comme le leader du marché, affiche plus de 650000 clients.

\section{La législation des tests (nutri)génétiques}

2004 : la loi de bioéthique interdit, en France, le recours aux tests ADN en dehors des domaines médical, scientifique et juridique.

2006 : la Federal Trade Commission invite les consommateurs américains à se méfier des tests génétiques en vente directe sur internet.

2013: la Food and Drug Administration interdit à 23andme la commercialisation des tests en vente directe sur internet.

2013: l'arrêté du 27 mai précise, en France, que le prescripteur de test génétique doit être un professionnel de santé ou un laboratoire agréé. influences des pratiques alimentaires sur l'expression des gènes (Castle et al., 2007 ; Ferguson, 2013). En tentant d'une part de comprendre comment le génome influence la manière dont un individu ou une population réagit à son alimentation et d'autre part d'expliquer de quelles manières les nutriments influencent l'expression du génome (d'un individu ou d'une population), ces grilles de lecture participent à l'articulation des échelles individuelle et populationnelle. Toutes deux renvoient au champ de la «nutrition personnalisée » qui invite les individus à adapter leurs pratiques alimentaires en fonction de leur patrimoine génétique et des facteurs de risque associés, et relativise l'intérêt des recommandations de santé publique basées sur une "one size fits all medicine». Ce champ émergeant est aujourd'hui relativement bien institutionnalisé, en témoignent la mise en place de réseaux scientifiques $(\mathrm{NuGO}$ - European Nutrigenomics Organisation en 2004), le lancement de programmes de recherche (Food4Me en 2006 dans le cadre du $7^{\mathrm{e}}$ programme-cadre de la Commission européenne) ou encore la création de revues à comité de lecture, dont les facteurs d'impact sont depuis croissants (Genes \& Nutrition en 2006, Journal of Nutrigenetics and Nutrigenomics en 2007). Concrètement, des entreprises - à l'intersection entre recherche biomédicale et marketing nutritionnel (Saukko et al., 2010) commercialisent depuis quelques années des tests nutrigénétiques accessibles par le biais de la relation médicale et/ou en vente directe sur internet (Tab. 2). En échange de quelques centaines de dollars et d'un peu de salive, elles envoient aux intéressés une liste des facteurs de risque auxquels ils seraient génétiquement exposés, liste accompagnée de recommandations nutritionnelles dites «personnalisées». Les premiers tests commercialisés étaient même agrémentés d'une suggestion de compléments alimentaires, les industries médicamenteuses et agroalimentaires suivant de près le développement du champ. À titre d'exemple, l'International Society of Nutrigenetics and Nutrigenomics, une société savante établie en 2005 et organisant depuis une conférence internationale annuelle sur le thème, associait à son comité scientifique de départ des chercheurs issus du monde académique et de l'industrie agroalimentaire. Ces tests, s'ils existent et occupent toujours une place dans l'espace marchand malgré l'interdiction faite en 2013 par la Food and Drug Administration (FDA) à 23andme (le leader du marché) de les commercialiser en ligne, demeurent toutefois très controversés, tant d'un point de vue scientifique ${ }^{6}$ que du point de vue des débats que suscitent les enjeux éthiques, législatifs et sociaux ${ }^{7}$ qu'ils soulèvent. De surcroît, le paradigme explicatif sur lequel ils reposent est à la fois décevant en termes d'applications pratiques et scientifiquement dépassé (en grande partie), notamment du fait des avancées en matière d'épigénétique.

\footnotetext{
${ }^{6}$ En 2006, un comité sénatorial américain (United States Government Accountability Office - US GAO) a par exemple rendu publics les résultats d'une enquête prouvant la fragilité scientifique des recommandations issues des premiers tests nutri-génétiques. Autre point sensible : l'application à l'échelle individuelle de la notion de «facteur de risque», qui renvoie à une approche épidémiologique. Voir Lupton (1995).

${ }^{7}$ Ces enjeux font référence au domaine de recherche des ELSI (Ethical, Legal and Social Issues) qui s'est développé dans le cadre du Projet Génome Humain. Pour un aperçu des ELSI soulevés par la nutri-génétique, voir Castle et Ries (2009).
} 


\section{La nutri-épigénétique: le tournant environnementaliste}

Si la présence ou l'absence de certains gènes peut influer sur le développement de certaines pathologies, ce niveau de connaissance ne rassemble pas toute l'information nécessaire à la compréhension du vivant (Morange, 2011 ; Biémont, 2008). Nombreuses sont les études qui ont démontré que des plantes ou animaux ayant le même code génétique se développent différemment en fonction de l'environnement dans lequel ils évoluent (Paldi, 2009). Les travaux menés sur des jumeaux monozygotes soulignent le même processus au sein de l'espèce humaine (Fraga et al., 2005). Le code génétique, dont un individu hérite à la naissance, serait un stock d'informations programmatiques dont la mise en œuvre et l'expression seraient influencées par l'environnement. Ni la formulation de l'interaction gène-environnement, ni l'idée même de l'existence du niveau épigénétique ${ }^{8}$ ne sont neuves. Mais des avancées empiriques permettent aujourd'hui de rentrer avec précision dans l'étude de ces influences. Au point que l'on parle désormais d'épigénétique environnementale (Jirtle et Skinner, 2007). Celle-ci postule que les gènes sont contrôlés par une série d'interrupteurs que l'environnement (nutritionnel, psycho-affectif, etc.) aurait le pouvoir d'activer ou de désactiver (Bird, 2007; Francis, 2011; Carey, 2012; Woodward et Gills, 2012). Ces influences sont appelées "marques épigénétiques» et prendraient la forme de méthylation de l'acide désoxyribonucléique $(\mathrm{ADN})$. Elles ne modifient pas les gènes eux-mêmes, mais seulement leur expression. On a un temps considéré qu'au moment de la fécondation (plus précisément au cours de l'étape de la méiose), il y avait un «nettoyage» des marques épigénétiques des parents. Cependant, des travaux expérimentaux sur la souris ont montré que les brins d'ADN portés par les gamètes (spermatozoïdes et ovules) ne sont pas totalement «vierges» de ces molécules de transcription (Junien, 2012). Aussi est-il aujourd'hui admis que les marques épigénétiques sont principalement perdues lors du passage d'une génération à la suivante, mais que certaines d'entre elles «échapperaient» aux phases de reprogrammation des génomes parentaux au cours desquelles elles sont censées être effacées (Heard et Martienssen, 2014) et pourraient donc être transmissibles d'une génération à l'autre. Le génome affiche une forte stabilité au cours de la vie d'un individu alors que l'épigénome (l'ensemble des marques épigénétiques) est, quant à lui, assez variable et évolue tout au long de la vie. Cette variabilité est illustrée dans la littérature par la potentielle réversibilité des marques épigénétiques, ce

\footnotetext{
${ }^{8}$ L'épigénétique, terme formulé pour la première fois en 1942 par Conrad Waddington, s'intéresse à ce qu'il se passe "sur » les gènes (épi signifiant «sur» en grec) et non à leur séquence.
}

qui ouvre de nouveaux horizons d'applications dans le domaine thérapeutique. Des travaux sur des modèles animaux permettent d'envisager le fait que certains facteurs environnementaux comme l'alimentation postnatale ou encore «l'affection maternelle ${ }^{9}$ pourraient déprogrammer certaines marques épigénétiques. Parmi les débats actuels, le transfert des connaissances acquises sur les modèles végétaux/animaux à l'espèce humaine, la validation de la transmission transgénérationnelle des marques épigénétiques et l'analyse du degré de leur «verrouillage» et du type d'influence des facteurs environnementaux (on/off vs continuum) sont au premier plan (Junien, 2012; Heard et Martienssen, 2014). Audelà de ces questions scientifiques, liées à la compréhension des phénomènes, il est une controverse relative à la nature des données et aux méthodologies de collecte qui partage la communauté scientifique. Elle porte sur l'intérêt et les modalités de l'épi-séquençage, c'est-à-dire sur le fait de séquencer l'épigénome afin de répertorier l'ensemble des marques épigénétiques, de les analyser et de les comparer. L'idée, lancée au lendemain de la fin du séquençage du génome humain, oppose deux camps. Le premier, représenté par des réseaux de recherche très institutionnalisés (comme le réseau d'excellence Epigénome financé par la Commission européenne ou le Roadmap Epigenomics Consortium qui émane des National Institutes of Health - NIH - aux États-Unis), travaille dans cette direction. L'autre camp, dont certains chercheurs en biologie et génétique interviewés dans le cadre de cette recherche, adopte une posture plus critique. Il évoque l'énorme dépense publique consacrée à cette nouvelle "prouesse technologique », dénonce une «course aux données» plutôt que la formulation de « vraies questions » et relativise son intérêt scientifique, mettant en avant la variabilité temporelle et contextuelle de l'épigénome et donc les difficultés d'interprétation de son séquençage.

$\mathrm{Ce}$ que cet article veut souligner est que «l'environnement alimentaire ${ }^{10}$ jouerait un rôle prépondérant dans le processus épigénétique, au point que certains proposent de parler de nutri-épigénétique

\footnotetext{
${ }^{9}$ Selon Ian Weaver et ses collaborateurs (2004), «l'affection maternelle » de rates envers leurs petits (léchage et toilettage) modifierait l'épigénome de ces derniers et améliorerait leur réponse au stress à l'âge adulte.

${ }^{10}$ Ce concept, proposé par Landecker (2011) pour saisir la manière dont l'alimentation devient un «environnement molécularisé », gagnerait à être élargi avec les acquis de la sociologie de l'alimentation. Nous proposons d'intégrer aux aspects nutritionnels classiques les dimensions de l'environnement dans lequel la consommation alimentaire s'organise : environnements physique (disponibilité alimentaire en termes spatiaux et géographiques), économique (accessibilité alimentaire) et social (degré de commensalité et niveau de dépendance alimentaire des individus).
} 
(Gallou-Kabani et al., 2007; Burdge et Lillycrop, 2010 ; Choi et Friso, 2010 ; Junien, 2015). Ingérés au quotidien, les nutriments pourraient bloquer ou activer l'expression des gènes tout au long de la vie d'un individu. Il existerait toutefois des fenêtres d'exposition critique. La période de la vie intra-utérine a un temps été considérée comme la principale et des hypothèses d'une influence à long terme sur le statut pondéral et la santé ont été avancées. Puis d'autres fenêtres sont apparues en amont et en aval de la grossesse. C'est en grande partie pour cela que les «mille premiers jours de vie» font aujourd'hui l'objet d'une attention particulière (Hochberg et al., 2011). Ce que mangent les parents durant la période périconceptionnelle, ce que mangent les femmes durant leur grossesse puis ce que mangent les enfants durant les deux premières années de leur vie influenceraient, par modification épigénétique, la santé future de ces derniers ${ }^{11}$. Concrètement, le comportement alimentaire durant ces mille jours ne déterminerait pas la survenue de maladies à l'âge adulte mais créerait une susceptibilité ou une résistance à développer, plus tard, une ou des pathologies chroniques (cancers, maladies cardiovasculaires, diabète, etc.) en fonction de l'évolution du contexte nutritionnel des individus. Cette susceptibilité, à la fois héritée et acquise, pourrait ensuite être transmise à la génération suivante ${ }^{12}$. C'est sur cette base qu'une campagne de sensibilisation au rôle de la nutrition intrautérine et infantile durant les «mille premiers jours de vie» a été lancée en 2010 sous le patronage d'Hilary Clinton et à destination des Suds marqués par des situations de sous- et malnutrition. Elle résulte d'une coalition entre des ONG spécialisées dans les domaines de l'aide humanitaire et de la malnutrition (notamment Gain et InterAction) et les gouvernements états-unien et irlandais, coalition qui débouche la même année sur la création de l'ONG Thousand Days financée en grande partie par la fondation Bill et Melinda Gates. Cette initiative, rapidement soutenue par l'Organisation mondiale de la santé (OMS), s'est depuis globalisée et s'applique désormais aux problématiques de mal- et surnutrition dans les Nords ${ }^{13}$.

\footnotetext{
${ }^{11}$ Période périconceptionnelle + grossesse + deux premières années de vie $=$ mille jours.

${ }^{12}$ L'Étude de la famine hollandaise de 1944 a montré que les enfants conçus durant cette période auraient davantage développé de maladies métaboliques à l'âge adulte que les enfants conçus un peu avant ou un peu après. De plus, cette susceptibilité se serait transmise de manière épigénétique durant trois générations. Voir Carey (2012).

${ }^{13}$ À l'heure où nous rédigeons cet article, la quatrième version du Programme national nutrition santé (PNNS) se prépare à intégrer un volet « 1000 jours» en France afin de sensibiliser les couples ayant un projet d'enfant, les femmes enceintes et les parents de jeunes enfants au rôle de la nutrition sur la santé future de ces derniers.
}

\section{Les enjeux liés à la personnalisation}

La GN se déploie dans un contexte social marqué par une forte injonction à être acteur de sa propre santé (Aïach et Delanoë, 1998 ; Peretti-Watel et Moatti, 2009; Fainzang, 2012) et par une médicalisation et «nutritionnalisation» de l'alimentation (Poulain, 2009). En même temps, se développe une crise des relations entre science et société (Matalon, 2003 ; Joly et al., 2012). En cela, la GN contribue à l'émergence d'une médecine personnalisée dans laquelle l'alimentation jouerait un rôle important tant dans la prévention que dans la prise en charge des pathologies non transmissibles (Fournier et al., 2011 ; Poulain, 2013) : évincement des médecins, individualisation des recommandations et empowerment des patients (Hedgecoe, 2004; Howard et Borry, 2012; Tutton, 2012). Dans la littérature, les enjeux éthiques portés par cette nouvelle forme de médecine se cristallisent en deux principaux points: d'une part le stockage, la circulation, l'utilisation et la communication des données et informations génétiques (Tutton et Prainsack, 2011; Rouvroy, 2007) et d'autre part la réactivation des débats sur l'eugénisme (Winkler, 2008; Haug et Poindron, 2008; Vailly, 2011). Nous souhaitons ici attirer l'attention sur deux autres enjeux qu'induit l'idée même de personnalisation: l'émergence de nouvelles formes de responsabilité aux échelles individuelle, intergénérationnelle et politique, et la perturbation des modes de régulation sociale de l'alimentation.

\section{Nouvelles formes de responsabilité sociale et politique}

La GN s'appuie principalement sur une logique préventive et porte une «promesse médicale» (Dalgalarrondo et Hauray, 2015). En affinant la connaissance des facteurs de risque et en proposant des recommandations personnalisées, elle promet aux individus une meilleure maîtrise de leur santé et une plus grande anticipation de leur avenir (et de celui de leur descendance). Cette technique de personnalisation introduit de nouvelles formes de responsabilité (Hervé et al., 2008), que nous identifions à trois niveaux. Tout d'abord à l'échelle de la vie individuelle, car les applications de la GN (notamment les tests nutrigénétiques) invitent les individus à être autonomes, responsables et anticipateurs en matière de santé, et contribuent ainsi au renforcement de «l'homo medicus » (Pinell, 1992; Peretti-Watel et Moatti, 2009). La technique de personnalisation s'inscrit dans la politique de responsabilisation inhérente au principe de prévention déployé dans les sociétés contemporaines.

Ensuite, à l'échelle intergénérationnelle, «l'initiative des mille jours» introduit une forte injonction au «soin alimentaire» (bien manger et cuisiner pour la santé 
future de son enfant). Cette nouvelle forme de responsabilité, dont le risque est le glissement vers une sur-responsabilisation, un sur-travail domestique et une culpabilisation quotidienne des parents et notamment des mères (Richardson et al., 2014 ; Gojard, 2010 ; Garcia, 2011; Fournier et al., 2015) ${ }^{14}$, introduit de nouveaux liens sociaux et sanitaires entre générations. La nutri-épigénétique pourrait ainsi révéler de manière rétrospective des liens entre les pathologies d'un individu et les comportements alimentaires de ses parents avant la conception ou durant la grossesse. Même si ces liens sont encore hypothétiques, leur dévoilement pourrait avoir un impact considérable sur les relations familiales et sociales. Ceci posant la question de la volonté de savoir et celle de la responsabilité d'actes individuels inscrits dans une époque et un contexte de connaissance qui ne permettaient pas d'envisager ce type de responsabilité. C'est donc une tension paradoxale entre l'individualisation des liens alimentation-santé et la responsabilité à l'égard des autres qui se met en place au niveau de la nutrition, l'alimentation et leurs conséquences.

Enfin, l'échelle politique prolonge cette tension, certains mécanismes épigénétiques pouvant engager la responsabilité des pouvoirs publics (Hedlund, 2012). $\mathrm{Si}$ une exposition répétée à un nutriment ou un contaminant alimentaire - Hannah Landecker (2011) parle d' " exposition alimentaire » - au début de la vie d'un individu conduit à une plus grande sensibilité quant au développement de pathologies chroniques à l'âge adulte et que cette sensibilité peut être transmise aux générations suivantes, alors des actions de santé publique apparaissent nécessaires. Si des actions «par l'environnement» semblent possibles et ont été évoquées à l'Assemblée Nationale fin $2015^{15}$ (évolution des normes sanitaires en matière d'agriculture, formation du personnel de la petite enfance, etc.), c'est pourtant le choix d'une politique publique « à moindres frais » qui s'est imposé : celle visant à sensibiliser les couples ayant un projet d'enfant, les femmes enceintes et les parents de jeunes enfants au rôle de la nutrition intra-utérine et infantile. Une telle politique, essentiellement basée sur la responsabilisation des individus, semble d'une part faire fi de l'existence et de

\footnotetext{
${ }^{14}$ Le statut nutritionnel des souris mâles au moment de la conception aurait une incidence, par mécanisme épigénétique, sur la santé future de leur descendance (Lambrot et al., 2013). Celui des hommes est aussi à considérer. Toutefois, le risque de sur-responsabilisation et de culpabilisation concerne plus les femmes, car la grossesse est l'une des fenêtres d'exposition les plus critiques et elles sont toujours surinvesties dans les tâches alimentaires domestiques (charges physique et mentale).

${ }^{15}$ Il s'agissait d'une audition publique à l'initiative de l'Office parlementaire d'évaluation des choix scientifiques et technologiques (OPECST) sur «les enjeux éthiques et sociétaux de l'épigénétique» qui s'est déroulée à Paris le 25 novembre 2015.
}

la possible reproduction d'inégalités sociales de santé et risque d'autre part de les renforcer (Loi et al., 2013; Guibet Lafaye, 2014).

\section{Articuler « fait nutritionnel » et « fait alimentaire »}

Dans l'hypothèse du développement des tests nutri-épi-génétiques, de leur démocratisation et d'une diffusion massive des connaissances issues de la nutriépigénétique, les processus de pharmaceuticalisation de la vie quotidienne (Fox et Ward, 2008) et d'individualisation, de médicalisation et de nutritionnalisation de l'alimentation (Korthals, 2011; Poulain, 2012; Nordström et al., 2013) à l'œuvre dans les sociétés contemporaines se trouveraient fortement accélérés. Trois conséquences de «l'érosion» des modèles alimentaires (Beardsworth, 1995) doivent selon nous être soulignées. La première, d'ordre moral, renvoie au risque de gommer les dimensions socioculturelles de l'alimentation et uniformiser les pratiques et représentations alimentaires. Quels en seraient les impacts en termes d'identités et de patrimoines? Si ce mouvement accentue la concurrence entre l'aspect nutritionnel et les autres horizons de l'acte alimentaire (Poulain, 2001; Fournier, 2014), pourquoi serait-il plus légitime de favoriser l'expansion du premier plutôt que le maintien des seconds? Et quel sens donner aux comportements "récalcitrants», aux conduites dites à risque? La deuxième conséquence est d'ordre sanitaire. Elle renvoie à l'idée que certaines des dimensions sociales de l'alimentation pourraient avoir une fonction régulatrice sur le plan nutritionnel (McIntosh, 1999; Sobal, 2000; Fischler, 2013). Effectivement, la commensalité ${ }^{16}$, de par son caractère normatif et le contrôle social qu'elle occasionne, constituerait un «garde-fou» nutritionnel (Fournier, 2012a). Certains émettent même l'hypothèse selon laquelle l'attachement au «manger ensemble» pourrait en partie expliquer le faible niveau de prévalence de l'obésité en France (Fischler, 2011). Les promesses de la nutri-génétique en termes de prévention nutritionnelle pourraient se révéler en partie contre-productives en ce qu'elles renforceraient l'individualisation du rapport à l'alimentation. La perturbation des modes de régulation sociale de l'alimentation pourrait alors favoriser la montée des troubles du comportement alimentaire. Enfin, la troisième conséquence touche à la question de la transmission, l'enjeu étant d'identifier les effets de couplage entre les étapes de vie alimentaire et les fenêtres d'exposition critique (celle des mille jours notamment). La littérature montre que le fait de devenir parent est associé à une plus grande perméabilité nutritionnelle (Corbeau, 2010). Avec l'arrivée d'un premier enfant, les

\footnotetext{
$\overline{{ }^{16} \text { Le fait de partager un }}$ repas.
} 
parents sont plus attentifs à l'équilibre alimentaire et à la composition nutritionnelle des repas. Une étude française réalisée auprès de pères et mères d'enfants de moins de trois ans (Bocquet et al., 2013) a toutefois montré que l'attention des parents sur l'alimentation de leur progéniture glisse rapidement de l'aspect nutritionnel à l'aspect socialisateur. Les enfants, une fois qu'ils mangent diversifié, doivent effectivement apprendre à consommer les aliments dans le cadre des règles sociales qui encadrent l'acte alimentaire de leur groupe: par exemple manger ensemble et à table. Si les enjeux de la transmission alimentaire peuvent correspondre aux préconisations issues de "l'initiative des mille jours» durant la première année de l'enfance, un décalage semble s'opérer pour la suite.

Le défi consiste alors à articuler le «fait nutritionnel», qui au fur et à mesure que les connaissances avanceront - par l'identification de plus en plus précise de facteurs personnels de risque - renforcera inéluctablement l'individualisation du rapport à l'alimentation, avec le «fait alimentaire», qui est un acte de partage s'inscrivant dans des cadres sociaux et culturels. «Fait nutritionnel » et « fait alimentaire », deux dimensions qui participent au bien-être et à la santé des mangeurs (Poulain, 2012). L'enjeu des années à venir sera donc de concilier la personnalisation des recommandations nutritionnelles et les dimensions collectives de l'alimentation.

\section{Repenser les liens alimentation-santé dans une perspective interdisciplinaire}

Au-delà des enjeux sociaux précédemment abordés, la GN pose des questions d'ordre épistémologique. Combinées, la nutri-génétique, la nutri-génomique et la nutri-épigénétique ouvrent de nouvelles échelles temporelles pour penser les liens alimentation-santé (Poulain, 2013). La première est celle des générations et l'on commence à décrypter l'influence des contextes alimentaires et des modèles de consommation sur le portefeuille de gènes dans lequel le «hasard» de la conception puise pour former le génome et l'épigénome des individus. La seconde, plus courte, est celle de la vie individuelle : il s'agit ici de s'intéresser à ce que les individus mangent au quotidien, à leur style de vie alimentaire et à l'influence de leur alimentation sur l'expression des gènes tout au long de leur vie. Enfin, vient le temps plus court encore de la transmission : l'alimentation des parents durant la période périconceptionnelle et celle des femmes enceintes pourraient favoriser ou bloquer l'expression de certains gènes des enfants de façon plus ou moins durable. Cette triple temporalité fait émerger de nouveaux champs de recherche empirique qui sont de nature à redessiner les frontières et la « répartition des tâches » entre sciences humaines et sociales et sciences de la vie.
La nutri-génétique renforcerait une logique de «généticisation》 (Lippman, 1992; Rose, 2007; Hervé et al., 2008) qui désigne, selon Antoinette Rouvroy (2008, p. 111), «la contamination progressive des discours sociétaux [...] par une logique réductionniste et essentialiste faisant des gènes la cause privilégiée sinon exclusive des variations inter-personnelles et intercommunautaires au sein de l'espèce humaine, et survalorisant le caractère prédictif $[\ldots]$ des facteurs génétiques au détriment des facteurs socioéconomiques et environnementaux ». Dans cette perspective, les sciences sociales ont peu de prise si ce n'est d'en étudier les enjeux dans une posture critique. La nutriépigénétique permet en revanche d'envisager des interactions entre les «facteurs génétiques» et les « facteurs socioéconomiques et environnementaux», et de poser la question suivante : comment le social agit-il sur le biologique? Dans un article interrogeant les connexions possibles entre sociologie et épigénétique, Hannah Landecker et Aaron Panofsky (2013, p. 346) invitent à considérer la proximité conceptuelle et la complémentarité des deux disciplines: «Epigenetic logic implies that social structure and social regulation are directly and causally linked to genome structure and gene regulation $\gg{ }^{17}$. L'alimentation - c'est-à-dire des pratiques, des valeurs, des interactions et des normes sociales et culturelles - activerait ou bloquerait l'expression de certains gènes. En retour, pourrait se créer une sensibilité ou une résistance à développer, plus tard (à l'échelle de la vie individuelle ou des générations), des pathologies chroniques. Dans cette interaction gènes-environnement, le génome n'est toutefois pas qu'un medium : des processus moléculaires complexes et dynamiques opèrent et «décident» ou non d'intégrer les effets de l'environnement (social). Cet aller-retour entre le social et le biologique est appelé «molecular conduit model» (Weaver et al., 2004). Dès lors, comment étudier ces interactions entre le social et le biologique, quels outils méthodologiques mobiliser? L'enjeu est qu'un nouveau type de dialogue basé sur des données empiriques pouvant jouer un rôle dans les deux espaces de détermination s'ouvre entre sociologues et biologistes/généticiens. Il conviendrait tout d'abord de comprendre comment des concepts d'apparence similaire sont définis de part et d'autre (déterminisme, interaction, régulation, structure, etc.) et de saisir ce que le social signifie depuis les sciences de la vie. Le développement de l'épigénétique est une invitation, comme le suggère un éditorial de la revue Nature ${ }^{18}$, à ce que les sciences sociales et les sciences de la vie enterrent

\footnotetext{
${ }^{17}$ «Selon la logique épigénétique, la structure sociale et la régulation sociale sont directement et causalement liées à la structure du génome et à la régulation des gènes » (traduction des auteurs).

${ }^{18}$ Nature Editorial Group, 2012. "Life stresses”, Nature, 490, 143.
} 
la hache de guerre. Car pour la première fois, des données empiriques susceptibles d'être reconnues par les deux camps scientifiques pourraient permettre de tracer l'influence des modes de vie sur le biologique. Se réouvre alors toute une série de questions sur la génétique des populations, à l'intersection entre biologie et culture (de Garine, 1994). Quels modes de vie contribuent à la sélection génétique? Quels comportements alimentaires influencent la santé des générations futures? Dans un texte princeps de l'anthropologie biologique, Jean Benoist (1991, p. 115) précisait que « la culture n'est pas en aval du biologique : elle est en amont d'une part importante de la mise en jeu des mécanismes biologiques de l'évolution et de la diversification de l'espèce $»$. À travers les habitudes alimentaires, le social et le culturel s'immiscent dans le biologique. Si le modèle bio-psycho-socio-culturel développé par Edgar Morin (1973) pour rendre compte de l'irréductible complexité des interactions nature-culture était jusque-là cantonné au statut d'essai philosophique, il pourrait trouver dans la nutri-épigénétique une base empirique susceptible de changer son statut épistémologique. La GN invite ainsi à repenser les liens alimentationsanté dans une perspective interdisciplinaire. Au même titre que pour les sciences de l'environnement, cette perspective devrait être aujourd'hui acceptée comme «attitude scientifique de base et comme présupposé méthodologique» (Leroy, 2004).

\section{Conclusion: retour sur la question de «l'autonomie du fait social»}

La (re)découverte et le développement de l'épigénétique, qui permet non seulement d'expliquer l'adaptation d'une espèce à son environnement mais fait également la démonstration que les comportements peuvent agir sur l'expression des gènes, sont, pour certains biologistes, la grande révolution scientifique de la décennie en cours. Un certain nombre d'enjeux sociaux, nous l'avons vu, accompagnent l'avancée de ces connaissances scientifiques. Ainsi, le développement de la GN intensifie la médicalisation et la nutritionnalisation de l'alimentation quotidienne, des tendances déjà à l'œuvre depuis plusieurs décennies dans les sociétés occidentales. Il crée par ailleurs les conditions du déploiement de nouvelles formes de responsabilité individuelle, sociale et politique.

Mais c'est aussi sur le plan épistémologique que ces avancées vont avoir des conséquences, la principale étant selon nous la réouverture du débat sur les formes d'interdisciplinarité. La production de données empiriques susceptibles d'opérer un pont entre des espaces épistémologiques jusque-là relativement autonomes peut effectivement redistribuer les cartes et redessiner les lignes de partage entre les sciences sociales, biomédicales et de la vie. À travers cela, ce sont les modalités du dialogue interdisciplinaire et la question de l'autonomie du social posée par Durkheim (1894), à l'aube de la discipline, qui sont interrogées. La sociologie contemporaine est partagée entre deux postures. La première s'inscrit dans le respect scrupuleux de ce principe d'autonomie et envisage, au mieux, une possible communication au sein des sciences humaines et sociales (Berthelot, 1999; Valade, 1999). La seconde s'inscrit dans la tradition incarnée par Marcel Mauss et envisage le dialogue entre les territoires scientifiques qui se trouvent de part et d'autre de la «grande muraille» qui sépare le monde des sciences de la vie et des sciences humaines et sociales (Morin, 1990; Fischler, 1990; Poulain, 2002). En permettant d'éviter l'écueil des postures surplombantes de l'essai philosophique, la mise au jour de données empiriques sur lesquelles fonder ce dialogue devrait donner un nouveau souffle à certains champs de la sociologie : l'alimentation mais également la santé, le genre, le corps, le vieillissement ou encore l'enfance.

\section{Références}

Aïach P., Delanoë D., 1998. L'ère de la médicalisation. Ecce homo sanitas, Paris, Economica.

Barnes B., Dupré J., 2008. Genomes and what to make of them, Chicago, University of Chicago Press.

Beardsworth A., 1995. The management of food ambivalence: Erosion or reconstruction?, in Maurer D., Sobal J. (Eds), Eating agendas. Food and nutrition as social problems, New York, Aldine de Gruyter, 117-143.

Benoist J., 1991. Anthropologie biologique. Le biologique et le social, in Bonte P., Izard M. (Eds), Dictionnaire de l'ethnologie et de l'anthropologie, Paris, PUF, 113-116.

Berthelot J.-M., 1996. Les vertus de l'incertitude, Paris, PUF.

Berthelot J-M., 1999. Présentation, Sociologie et Sociétés, numéro spécial «La sociologie et les sciences sociales : une affaire de discipline(s) ?», 31, 1, 3-10.

Biémont C., 2008. Les éléments transposables: une force évolutive majeure, Natures Sciences Sociétés, 16, 1, 41-43.

Bird A., 2007. Perceptions of epigenetics, Nature, 447, 7143, 396-398.

Bocquet A., Corbeau J-P., Bocquet M., 2013. Étude Nutri-bébé SFAE 2013. Comportements alimentaires avant l'âge de 3 ans, Archives de Pédiatrie, 21, 5, 334.

Burdge G.C., Lillycrop K.A., 2010. Nutrition, epigenetics, and developmental plasticity: Implications for understanding human disease, Annual Review of Nutrition, 30, 1, 315-339.

Carey N., 2012. The epigenetics revolution. How modern biology is rewriting our understanding of genetics, disease, and inheritance, New York, Columbia Univ. Press.

Castle D., Cline C., Daar A.S., Tsamis C., Singe P.A., 2007. Science, society, and the supermarket. The opportunities and challenges of nutrigenomics, Wiley, Hoboken.

Castle D., Ries N. (Eds.), 2009. Nutrition and genomics. Issues of ethics, law, regulation and communication, New York, Academic Press. 
Choi S.W., Friso S., 2010. Epigenetics: A new bridge between nutrition and health, Advances in Nutrition, 1, 1, 8-16.

Corbeau J-P. (Dir.), 2010. Trajectoires alimentaires et parcours de vie, Paris, INPES.

Dalgalarrondo S., Hauray B., 2015. Les économies de la promesse anti-âge. Le cas de la DHEA, Sciences Sociales et Santé, 33, 2, 5-30.

Dupuy A., 2014, Thématisation du plaisir alimentaire et visées utilitaristes, Sociologie et sociétés, 46, 2, 253-275.

Durkheim E., 1894. Les règles de la méthode sociologique, Paris, PUF.

Fainzang S., 2012. L'automédication ou les mirages de l'autonomie, Paris, PUF.

Ferguson L.R. (Ed.), 2013. Nutrigenomics and nutrigenetics in functional foods and personalized nutrition, Boca Raton, CRC Press.

Fischler C., 1990. L'homnivore, Paris, Odile Jacob.

Fischler C., 2011. Commensality, society and culture, Social Science Information, 50, 3-4, 528-548.

Fischler C., 2013. Les alimentations particulières. Mangeronsnous encore ensemble demain?, Paris, Odile Jacob.

Fournier T., 2012a. Suivre ou s'écarter de la prescription diététique. Les effets du «manger ensemble» et du «vivre ensemble » chez des personnes hypercholestérolémiques en France, Sciences Sociales et Santé, 30, 2, 35-60.

Fournier T., 2012b. La sociologie face aux controverses médicales, Sciences Sociales et Santé, 30, 3, 115-119.

Fournier T., 2014. Face à l'injonction diététique, un «relativisme nutritionnel» en France, SociologieS, Premiers textes, https://sociologies.revues.org/4628.

Fournier T., Bruckert E., Czernichow S., Paulmyer A., Poulain J-P., 2011. The THEMA study: A sociodemographic survey of hypercholesterolaemic individuals, Journal of Human Nutrition and Dietetics, 24, 6, 572-581.

Fournier T., Jarty J., Lapeyre N., Touraille P., 2015. Alimentation, arme du genre, Journal des Anthropologues, 140-141, $19-49$.

Fox N.J., Ward K.J., 2008. Pharma in the bedroom.. and the kitchen.. The pharmaceuticalisation of daily life, Sociology of Health \& Illness, 30, 6, 856-868.

Fraga M.F., Ballestar E., Paz M.F., Ropero S., Setien F., Ballestar M., Heine-Suner D., Cigudosa J.C., Urioste M., Benitez J., Boix-Chornet M., Sanchez-Aguilera A., Ling C., Carlsson E., Poulsen P., Vaag A., Stephen Z., Spector T.D., Wu Y-Z., Plass C., Esteller M., 2005. Epigenetic differences arise during the lifetime of monozygotic twins, Proceedings of the National Academy of Sciences, 102, 30, 1060410609.

Francis R., 2011. Epigenetics: The ultimate mystery of inheritance, New York, W.W. Norton.

Gallou-Kabani C., Vigé A., Gross M.S., Junien C., 2007. Nutri-epigenomics: Lifelong remodelling of our epigenomes by nutritional and metabolic factors and beyond, Clinical Chemistry and Laboratory Medicine, 45, 3, 321-327.

Garcia S., 2011, Mères sous influence. De la cause des femmes à la cause des enfants, Paris, La Découverte.
Garine (de) I., 1994. The diet and nutrition of human populations, in Ingold T. (Ed.), Companion encyclopaedia of anthropology. Humanity, culture and social life, London, Routledge, 226-264.

Gojard S., 2010, Le métier de mère, Paris, la Dispute.

Guibet Lafaye C., 2014. L'épigénétique: pour de nouvelles politiques de santé? Humanistyka i Przyrodoznawstwo, 4-22.

Haug M., Poindron P., 2008. Les biotechnologies appliquées à la santé humaine: quelques enjeux sociaux, socioéconomiques, politiques et moraux, Revue des Sciences Sociales, 39, 72-81.

Heard E., Martienssen R.A., 2014. "Transgenerational epigenetic inheritance: Myths and mechanisms", Cell, 157, 1, 95-109.

Hedgecoe A., 2004. The politics of personalised medicine: Pharmacogenetics in the clinic, New York, Cambridge University Press.

Hedlund M., 2012. Epigenetic responsibility, Medicine Studies, 3, 3, 171-183.

Hervé C., Jean M.S., Molinari P.A., Grimaud M-A., Laforêt E. (Dir.), 2008. Généticisation et responsabilités, Paris, Dalloz.

Hochberg Z., Feil R., Constancia M., Fraga M., Junien C., Carel J., Boileau P., Le Bouc Y., Deal C., Lillycrop K., Scharfmann R., Sheppard A., Skinner M., Szyf M., Waterland R., Waxman D., Whitelaw E., Ong K., Albertsson-Wikland K., 2011. Child health, developmental plasticity, and epigenetic programming, Endocrine Reviews, 32, 2, 159-224.

Holton G. 1982. L'invention scientifique: thémata et interprétation, Paris, PUF.

Howard H.C., Borry P., 2012. Is there a doctor in the house? The presence of physicians in the direct-to-consumer genetic testing context, Journal of Community Genetics, 3 , 2, 105-112.

Jirtle R.L., Skinner M.K., 2007. Environmental epigenomics and disease susceptibility, Nature Reviews Genetics, 8, 4, 253-262.

Joly P-B., Martinand J-L., Millier C., 2012. Sciences / société : une relation évincée ?, Natures Sciences Sociétés, 20, 4, 393-394.

Junien C. 2012. L'épigénétique : les gènes et l'environnement, pour le meilleur et pour le pire, in Toussaint J-F., Swynghedauw B., Bœuf G. (Dir.), L'Homme peut-il s'adapter? Marges d'adaptation de l'espèce humaine face aux changements environnementaux, Versailles, Quae, 48-56.

Junien C., 2015. Alimentation et épigénétique, in Esnouf C., Fioramonti J., Laurioux B. (Eds), L'alimentation à découvert, Paris, CNRS Editions, 182-183.

Korthals M., 2011. Coevolution of nutrigenomics and society: Ethical considerations, American Journal of Clinical Nutrition, 94, 6, 2025-2029.

Lambrot R., Xu C., Saint-Phar S., Chountalos G., Cohen T., Paquet M., Suderman M., Hallett M., Kimmins S., 2013. Low paternal dietary folate alters the mouse sperm 
epigenome and is associated with negative pregnancy outcomes, Nature Communications, 4, 2889, 1-13.

Landecker H., 2011. Food as exposure: Nutritional epigenetics and the new metabolism, BioSocieties, 6, 2, 167-194.

Landecker H., Panofsky A., 2013. From social structure to gene regulation, and back: A critical introduction to environmental epigenetics for sociology, Annual Review of Sociology, 39, 333-357.

Lepiller O., 2012. Naturel, in Poulain J-P. (Dir.), Dictionnaire des cultures alimentaires, Paris, PUF, 905-911.

Leroy P., 2004. Sciences environnementales et interdisciplinarité : une réflexion partant des débats aux Pays-Bas, Natures Sciences Sociétés, 12, 3, 274-284.

Lippman A., 1992. Led (astray) by genetic maps: The cartography of the human genome and health care, Social Science and Medicine, 35, 12, 1469-1476.

Loi M., Del Savio L., Stupka E., 2013. Social epigenetics and equality of opportunity, Public Health Ethics, 6, 2, 142-153.

Lupton D., 1995. The imperative of health. Public health and the regulated body, London, Sage.

Matalon B., 2003. Crise de la science ou crise de la société ?, Les Cahiers de Psychologie Politique, 4, http://lodel. irevues.inist.fr/cahierspsychologiepolitique/index.php?id= 1373.

McIntosh W.A., 1999. The family meal and its significance in global times, in Grew R. (Ed.), Food in global history, Boulder, Westview Press, 217-239.

Morange M., 2011. La vie, l'évolution et l'histoire, Paris, Odile Jacob.

Morin E., 1973. Le paradigme perdu: la nature humaine, Paris, Seuil.

Morin E., 1990. Sur l'interdisciplinarité, in Carrefour des sciences, Actes du colloque du Comité National de la Recherche Scientifique Interdisciplinarité, Paris, Editions du CNRS.

Nordström K., Coff C., Jonsson H., Nordenfelt L., Gorman U., 2013. "Food and health: individual, cultural, or scientific matters?", Genes \& Nutrition, 8, 4, 357-363.

Paldi A., 2009. L'hérédité sans gènes, Paris, Le Pommier.

Peretti-Watel P., Moatti J-P., 2009. Le principe de prévention. Le culte de la santé et ses dérives, Paris, Seuil.

Pinell P., 1992. Naissance d'un fléau. Histoire de la lutte contre le cancer en France (1890-1940), Paris, Métailié.

Poulain J-P., 2001. Manger aujourd'hui. Attitudes, normes et pratiques, Toulouse, Privat.

Poulain J-P., 2002. Sociologies de l'alimentation, Paris, PUF.

Poulain J-P., 2009. Sociologie de l'obésité, Paris, PUF.

Poulain J-P. (Dir.), 2012. Dictionnaire des cultures alimentaires, Paris, PUF.

Poulain J-P., 2013. Affirmation des particularismes individuels et évolution des modèles alimentaires, in Fischler C. (Ed.),
Les alimentations particulières. Mangerons-nous encore ensemble demain?, Paris, Odile Jacob, 248-259.

Poulain J-P., 2017. Socio-anthropologie du «fait alimentaire» ou food studies. Les deux chemins d'une thématisation scientifique, L’Année Sociologique, 67, 1, 23-45.

Prud'homme J., Gingras Y., 2015. Les collaborations interdisciplinaires: raisons et obstacles, Actes de la Recherche en Sciences Sociales, 210, 5, 40-49.

Richardson S., Daniels C.R., Gillman L.W., Golden J., Kukla R., Kusawa C., Rich-Edwards J., 2014. Don't blame the mothers, Nature, 512, 7513, 131-132.

Rose N., 2007. The politics of life itself: Biomedicine, power, and subjectivity in the twenty-first century Princeton, Princeton University Press.

Rouvroy A., 2007. Human genes and neoliberal governance. A foucaldian critique, Abingdon, Routledge.

Rouvroy A., 2008. Généticisation et responsabilité : les habits neufs de la gouvernance néolibérale, in Hervé C. Jean M.S., Molinari P.A., Grimaud M-A., Laforêt E. (Dir.), Généticisation et responsabilités, Paris, Dalloz, 111-120.

Saukko P.M., Reed M., Britten N., Hogarth S., 2010. Negotiating the boundary between medicine and consumer culture: Online marketing of nutrigenetic tests, Social Science and Medicine, 70, 5, 744-753.

Sobal J., 2000. Sociability and meals: Facilitation, commensality, and interaction, in Meiselman H.L. (Ed.), Dimensions of the meal: The science, culture, business, and art of eating, Gaithersburg, Aspen Publishers, 119-133.

Terssac (de) G., Lalande K., 2002. Du train à vapeur au TGV : sociologie du travail d'organisation, Paris, PUF.

Tutton R., 2012. Personalizing medicine: Futures, present and past, Social Science and Medicine, 75, 10, 1721-1728.

Tutton R., Prainsack, B., 2011. Enterprising or altruistic selves? Making up research subjects in genetics research, Sociology of Health and Illness, 33, 7, 1081-1095.

Vailly J., 2011. Naissance d'une politique de la génétique. Dépistage, biomédecine, enjeux sociaux, Paris, PUF.

Valade B., 1999. Le « sujet» de l'interdisciplinarité, Sociologie et Sociétés, numéro spécial «La sociologie et les sciences sociales : une affaire de discipline(s)? », 31, 1, 11-21.

Vinck D., 2000. Pratiques de l'interdisciplinarité, Grenoble, PUG.

Weaver I.C.G., Cervoni N., Champagne F.A., D'Alessio A.C., Sharma S., Seckl J.R., Dymov S., Szyf M., Meaney M.J., 2004. Epigenetic programming by maternal behavior, Nature Neuroscience, 7, 8, 847-54.

Winkler J.T., 2008. Imminent applications of nutrigenomics: A stakeholder analysis, in Kok F., Bouwman L., Desiere F. (Eds.), Personalized nutrition. Principles and applications, Boca Raton, CRC Press, 151-168.

Woodward T.E., Gills J-P., 2012. The mysterious epigenome: What lies beyond DNA, Grand Rapids, MI, Kregel.

Citation de l'article : Fournier T., Poulain J.-P., 2017. La génomique nutritionnelle : (re)penser les liens alimentation-santé à l'articulation des sciences sociales, biomédicales et de la vie. Nat. Sci. Soc. 25, 2, 111-121. 